\title{
LOCALLY FLAT STRINGS AND HALF-STRINGS ${ }^{1}$
}

\author{
C. LACHER ${ }^{2}$
}

1. Definitions. $R^{n}$ denotes euclidean $n$-space, $H^{n}=R^{n-1} \times[0, \infty)$ $C R^{n}, B^{n}$ is the unit ball in $R^{n}$, and $S^{n}$ is the one-point compactification of $R^{n}$. An $n$-string, $n$-half-string, $n$-cell, $n$-sphere, is a set which is homeomorphic to $R^{n}, H^{n}, B^{n}, S^{n}$ respectively. An $n$-manifold is a space $M$ such that each point of $M$ has a neighborhood homeomorphic to $R^{n}$; an $n$-manifold with boundary is a space each point of which has a neighborhood whose closure is an $n$-cell. If $M$ is an $n$-manifold with boundary, the set of points of $M$ which have neighborhoods homeomorphic to $R^{n}$ is denoted by $\dot{M}$ (the interior of $M$ ) and $M-\stackrel{M}{M}$ is denoted by $\dot{M}$ (the boundary of $M$ ). Let $M$ be a $k$-manifold with boundary contained in the $n$-manifold $N ; M$ is locally flat at the point $p \in \stackrel{\circ}{ }$ if there is a neighborhood $U$ of $p$ in $N$ such that $(U, U \cap M)$ is homeomorphic to the pair $\left(R^{n}, R^{k}\right) ; M$ is locally flat at the point $p \in \dot{M}$ if there is a neighborhood $U$ of $p$ in $N$ such that $(U, U \cap M$ ) is homeomorphic to $\left(R^{n}, H^{k}\right)$. The symbol $\approx$ will be used to mean "is homeomorphic to."

\section{Statement of results.}

Theorem 2.1. Let $X$ be a locally flat $k$-half-string in the $n$-manifold $M, k \leqq n$. Then there is an open set $U$ in $M$ containing $X$ such that $(U, X) \approx\left(R^{n}, H^{k}\right)$.

Theorem 2.2. Let $X$ be a locally flat $k$-string in the $n$-manifold $M$, $k<n$. Then there is an open set $U$ in $M$ containing $X$ such that $(U, X)$ $\approx\left(R^{n}, R^{k}\right)$.

Theorem 2.3. Let $X$ be a closed, locally flat $k$-half-string in $R^{n}$, $k \leqq n, n>3$. Then $\left(R^{n}, X\right) \approx\left(R^{n}, H^{k}\right)$.

Corollary 2.4. Let $Q$ be a $k$-cell in $S^{n}, k \leqq n, n>3$, and suppose that $Q-p$ is locally flat, where $p \in \dot{Q}$. Then $\left(S^{n}, Q\right) \approx\left(S^{n}, B^{k}\right)$.

COROLlaRy 2.5. Let $M$ be a k-manifold with boundary contained in the $n$-manifold $N, k \leqq n, n>3$. Suppose that $\stackrel{M}{M}$ is locally flat in $N$, and

Presented to the Society, January 25, 1966 under the title Almost locally flat n-cells; received by the editors December 16, 1965 .

1 This research constitutes a part of the author's doctoral dissertation under the direction of Professor J. C. Cantrell at the University of Georgia.

2 The author is an NSF Graduate Fellow. 
denote by $E$ the set of points of $\dot{M}$ at which $M$ fails to be locally flat. Then $E$ does not contain an isolated point, and hence $E$ is either empty or uncountable.

The following corollary gives the solution to a problem proposed by R. H. Fox ([4, Problem 21]).

Corollary 2.6. If $n>3$ and if $D$ is a wild $(n-2)$-cell in $R^{n}$ such that $D-p$ is locally flat for some point $p$, then $p$ must be an interior point of $D$, and $D$ must be a "mildly wild" cell in $R^{n}$; i.e., if $E$ is a cell which is locally flat in $D$ and which contains $p$ on its boundary then $E$ is tame in $R^{n}$.

For an example of a cell satisfying the hypothesis of Corollary 2.6 see [6].

Special cases of some of these results are known. Theorem 2.1 (with $k=n$ ) is proved in [1], Theorem 2.2, (with $k=n-1$ ) and Theorem 2.3 (with $k=n$ ) follow from the work in [3]; and Theorem 2.2 (with $k \leqq n-3$ ) follows from [5]. The three dimensional analogue of Theorem 2.3 is known to be false.

3. Preliminary lemmas. Let $M$ be an $n$-manifold and let $X$ be a locally flat $k$-half-string in $M, k \leqq n$. It is well known that locally flat cells in $R^{n}$ are trivially embedded in $R^{n}$. The proof of this result carries over to yield the following lemma, since any compact subset of $X$ is contained in a locally flat $k$-cell in $X$. (See $\S 6$.)

Lemмa 3.1. If $K$ is a compact subset of $X$ then there is an open set $U$ in $M$ such that $K \subset U$ and $(U, U \cap X) \approx\left(R^{n}, H^{k}\right)$.

Lemma 3.2. Let $U, V$ be open sets in $M$ such that $(U, U \cap X)$ $\approx\left(R^{n}, H^{k}\right) \approx(V, V \cap X)$ and $(U \cap X) \subset(V \cap X)$. If $C$ is a compact subset of $U$ then there is a homeomorphism $g$ of $M$ onto itself such that

(1) $g \mid X=$ identity,

(2) $g \mid M-U=i d e n t i t y$, and

(3) $g(V)$ contains $C$.

Proof. Let $W=\phi(U \cap V)$, where $\phi:(U, U \cap X) \approx\left(R^{n}, H^{k}\right) . W$ is an open subset of $R^{n}$ containing $H^{k}$. Let $f$ be a homeomorphism of $R^{n}$ onto itself which is the identity on $H^{k}$ and outside of some compact set and such that $f(W)$ contains $\phi(C)$. Then define $g$ on $M$ by $g=\phi^{-1} f \phi$ on $U$ and $g=$ identity otherwise.

Lemma 3.3. There exists a sequence $V_{1}, V_{2}, \cdots$ of open subsets of $M$ such that

(1) $\left(V_{i}, V_{i} \cap X\right) \approx\left(R^{n}, H^{k}\right)$ for each $i$; 
(2) $\bar{V}_{i} \subset V_{i+1}$ for each $i$; and

(3) $X \subset \bigcup_{i=1}^{\infty} V_{i}$.

Proof. Let $K_{1}, K_{2}, \cdots$ be a sequence of compact sets whose union is $X$, and let $U_{1}, U_{2}, \cdots$ be a sequence of open subsets of $M$ such that $\left(U_{i}, U_{i} \cap X\right) \approx\left(R^{n}, H^{k}\right), K_{i} \subset U_{i}$, and $\left(U_{i} \cap X\right) \subset\left(U_{i+1} \cap X\right)$ for each $i$. Let $h_{i}:\left(U_{i}, U_{i} \cap X\right) \approx\left(R^{n}, H^{k}\right)$ be a particular homeomorphism. The existence of the $U_{i}, h_{i}$ follows directly from Lemma 3.1. We shall apply Lemma 3.2 recursively on the $U_{i}$.

Step 1. Apply Lemma 3.2 with $U=U_{1}, V=U_{2}$, and $C=C_{1}=h_{1}^{-1}\left(B_{1}\right)$ $\cup K_{1}$, where $B_{1}$ is a (round) ball in $R^{n}$ centered at the origin. Let $g=g_{2}$ be given by $3.2, \tilde{U}_{2}=g_{2}\left(U_{2}\right)$, and $\tilde{h}_{2}=h_{2} g_{2}^{-1}$. Note that $\tilde{h}_{2}$ maps the pair $\left(\widetilde{U}_{2}, \widetilde{U}_{2} \cap X\right)$ homeomorphically onto $\left(R^{n}, H^{k}\right)$ and that $\widetilde{U}_{2}$ contains $h_{1}^{-1}\left(B_{1}\right) \cup K_{1}$.

Step 2. Apply Lemma 3.2 again, with $U=\widetilde{U}_{2}, V=U_{3}$, and $C=C_{2}$ $=\tilde{h}_{2}^{-1}\left(B_{2}\right) \cup K_{2}$, where $B_{2}$ is a ball in $R^{n}$ centered at the origin so that $\tilde{h}_{2}\left(C_{1}\right) \subset \ddot{B}_{2}$. Let $g=g_{3}$ be given by $3.2, \widetilde{U}_{3}=g_{3}\left(U_{3}\right)$, and $\tilde{h}_{3}=h_{3} g_{3}^{-1}$. Then $h_{3}$ maps $\left(\widetilde{U}_{3}, \widetilde{U}_{3} \cap X\right)$ homeomorphically onto $\left(R^{n}, H^{k}\right)$ and $\tilde{U}_{3}$ contains $\tilde{h}_{2}^{-1}\left(B_{2}\right) \cup K_{2}$.

Continuing this process, we get a sequence $\widetilde{U}_{1}, \widetilde{U}_{2}, \cdots$ of open sets in $M$ and a sequence $\tilde{h}_{1}, \tilde{h}_{2}, \ldots$ of homeomorphisms such that

(a) $\tilde{h}_{i}:\left(\tilde{U}_{i}, \tilde{U}_{i} \cap X\right) \approx\left(R^{n}, H^{k}\right)$ for all $i$;

(b) $\tilde{h}_{i+1}^{-1}\left(\stackrel{B}{i+1}_{i+1}\right) \supset \tilde{h}_{i}^{-1}\left(B_{i}\right)$ for all $i$; and

(c) $\tilde{h}_{i+1}^{-1}\left(B_{i+1}\right) \supset K_{i}$ for all $i$.

Define $V_{i}=\tilde{h}_{i}^{-1}\left(\dot{B}_{i}\right), i=1,2, \cdots$. Since $B_{i}$ is an open (round) ball centered at the origin in $R^{n}$, condition (1) follows from (a). Conditions (2) and (3) follow from (b) and (c) respectively, and the lemma is proved.

4. More lemmas. Let $M$ be an $n$-manifold and let $X$ be a locally flat $k$-string in $M, k<n$. For $i=1,2,3$, Lemma $4 . i$ is the same as Lemma 3.i except that $H^{k}$ in $3 . i$ is replaced by $R^{k}$ in $4 . i$. Otherwise, the statement and proof of $4 . i$ is identical with that of 3.i.

5. Proofs of theorems. We give the proof of Theorem 2.1. Theorem 2.2 follows from $\$ 4$ in exactly the same way that Theorem 2.1 follows from $\S 3$.

Proof of Theorem 2.1. (This proof is similar to one given in [2]; however, for the sake of completeness, the following modification is given.) Let $X$ be a locally flat $k$-half-string in the $n$-manifold $M$, and let $V_{1}, V_{2}, \ldots$ be a sequence of open subsets of $M$ as given by Lemma 3.3. We may assume that each $V_{i}$ has compact closure.

Sequences $Q_{1}, Q_{2}, \ldots$ of $n$-cells in $M$ and $g_{1}, g_{2}, \cdots$ of embeddings 
$g_{i}:\left(Q_{i}, Q_{i} \cap X\right) \rightarrow\left(R^{n}, H^{k}\right)$ will be constructed so that

(a) $Q_{i} \subset Q_{i+1}$ for each $i$;

(b) $g_{i+1} \mid Q_{i}=g_{i}$ for each $i$;

(c) $\bigcup_{i=1}^{\infty} Q_{i}=\bigcup_{i=1}^{\infty} V_{i} ;$ and

(d) $\bigcup_{i=1}^{\infty} g_{i}\left(Q_{i}\right)=R^{n}$.

Then we may define $U=\bigcup_{i=1}^{\infty} Q_{i}$ and $g=\bigcup_{i=1}^{\infty} g_{i}$ so that $g:(U, X)$ $\approx\left(R^{n}, H^{k}\right)$ is a homeomorphism.

For each $t>0, B_{t}$ denotes the ball with center 0 and radius $t$ in $R^{n}$. Let $Q_{1}=h_{1}^{-1}\left(B_{1}\right), g_{1}=h_{1} \mid Q_{1}$, and consider $h_{2} h_{1}^{-1}\left(B_{2}\right) \subset R^{n}$. Note that $h_{2}\left(\bar{V}_{1}\right)$ is a compact subset of $R^{n}$, so that there is a homeomorphism $\phi_{2}: R^{n} \approx R^{n}$ such that $\phi_{2}=$ identity on $h_{2} h_{1}^{-1}\left(B_{1}\right), \phi_{2}=$ identity outside some compact set in $R^{n}, \phi_{2}\left(H^{k}\right)=H^{k}$, and $\phi_{2}\left(h_{2} h_{1}^{-1}\left(B_{2}\right)\right)$ contains $h_{2}\left(\bar{V}_{1}\right)$. (See next paragraph for a construction of $\phi_{2}$.) Then define $Q_{2}=h_{2}^{-1} \phi_{2} h_{2} h_{1}^{-1}\left(B_{2}\right)$ and $g_{2}=h_{1} h_{2}^{-1} \phi_{2}^{-1} h_{2} \mid Q_{2}$. Notice that $g_{2} \mid Q_{1}$ $=h_{1} h_{2}^{-1} \phi_{2}^{-1} h_{2} h_{1}^{-1}\left|B_{1}=h_{1}\right| Q_{1}=g_{1}, Q_{1} \subset Q_{2}, \quad V_{1} \subset Q_{2}$, and $g_{2}\left(Q_{2}, Q_{2} \cap X\right)$ $=\left(B_{2}, B_{2} \cap H^{k}\right)$.

The homeomorphism $\phi_{2}$ may be obtained as follows. Let $A$ be a ball with center $h_{2} h_{1}^{-1}(0)$ such that $A \subset h_{2} h_{1}^{-1}\left(\stackrel{\circ}{B}_{1}\right)$. Then there is a homeomorphism $\psi: R^{n} \approx R^{n}$ such that $\psi=$ identity outside of $B_{2}$, $\psi\left(H^{k}\right)=H^{k}$, and $\psi\left(B_{1}\right) \subset h_{1} h_{2}^{-1}(\AA)$, and there is a homeomorphism $\tilde{\psi}: R^{n} \approx R^{n}$ such that $\tilde{\psi}=$ identity outside some compact set, $\tilde{\psi}=$ identity on $A, \tilde{\psi}\left(H^{k}\right)=H^{k}$, and $\tilde{\psi}\left(h_{2} h_{1}^{-1}\left(B_{2}\right)\right)$ contains $h_{2}\left(\bar{V}_{1}\right)$. Both $\psi$ and $\tilde{\psi}$ are homeomorphisms which map each half-ray emanating from the origin onto itself. Then $\phi_{2}$ may be defined by

$$
\phi_{2}=\left\{\begin{array}{l}
h_{2} h_{1}^{-1} \psi^{-1} h_{1} h_{2}^{-1} \tilde{\psi} h_{2} h_{1}^{-1} \psi h_{1} h_{2}^{-1} \text { on } h_{2} h_{1}^{-1}\left(B_{2}\right), \\
\psi \quad \text { outside of } h_{2} h_{1}^{-1}\left(B_{2}\right) .
\end{array}\right.
$$

Continuing in this way, the sequences $Q_{1}, Q_{2}, \cdots$ and $g_{1}, g_{2}, \cdots$ may be constructed, and the proof is complete.

Proof of Theorem 2.3. Let $X$ be a closed, locally flat $k$-half-string in $R^{n}, n>3$. Theorem 2.1 supplies a neighborhood $U$ of $X$ in $R^{n}$ and a homeomorphism $h:(U, X) \approx\left(R^{n}, H^{k}\right)$. Suppose that $Y$ is a locally flat, closed, $(n-1)$-string in $R^{n}$ such that $Y \cap H^{k}=\varnothing$ and such that $h^{-1}(Y)$ is closed in $R^{n}$. It is known [3] that locally flat, closed, $(n-1)$ strings in $R^{n}$ are trivially embedded if $n>3$; thus, if we can find $Y$, and if $V$ is the complementary domain of $Y$ which contains $H^{k}$, then the homeomorphism $g=h \mid h^{-1}(\bar{V})$ can be extended to a homeomorphism $\left(R^{n}, X\right) \approx\left(R^{n}, H^{k}\right)$.

To complete the proof, $Y$ must be constructed. If $B_{t}$ is the ball of radius $t$ and center 0 in $R^{n}, \quad h^{-1} \mid B_{t}$ is uniformly continuous for each $t$. Choose $\epsilon_{i}>0$ so that any set of diameter less than $\epsilon_{i}$ in $B_{i}$ is mapped 
by $h^{-1}$ onto a set of diameter less than 1 . Let $Y$ be a closed, locally flat $(n-1)$-string in $R^{n}$ such that $Y \cap H^{k}=\varnothing$ and such that distance $\left(y, H^{k} \cap\left(B_{i}-\stackrel{B}{B}_{i-1}\right)\right)<\epsilon_{i}$ for each $y \in Y \cap\left(B_{i}-\ddot{B}_{i-1}\right), i=1,2, \cdots$. It is then clear that a sequence $\left\{y_{j}\right\} \subset Y$ tends to infinity if and only if $\left\{h^{-1}\left(y_{j}\right)\right\}$ tends to infinity, so that $h^{-1}(Y)$ is closed in $R^{n}$. This completes the proof of 2.3 .

Corollary 2.4 follows immediately from 2.3. To prove Corollary 2.5 , use 2.4 to prove that a $k$-cell in $R^{n}, n>3$, may not fail to be locally flat at precisely one point if that point is a boundary point, and then apply this result locally to a supposed isolated point of $E$.

6. Appendix. The referee has pointed out that there is no proof of the statement "Locally flat cells in $R^{n}$ are trivially embedded in $R^{n}$ " available in the literature. The following lemma yields a proof of this statement via the generalized Schoenflies theorem. Moreover, Lemmas 3.1 and 4.1 of this paper follow directly from this lemma. The argument below was indicated to me first by Prabir Roy who attributed it to a seminar at the University of Wisconsin.

Lemma. Let $D$ be a locally flat $k$-cell in the $n$-manifold $M$. Then $D$ has a neighborhood $U$ in $M$ such that $(U, D) \approx\left(R^{n}, B^{k}\right)$.

Proof. Since $D$ is locally flat, there are open sets $U_{1}, \cdots, U_{s}$ in $M$ such that $D$ is covered by the $U_{i}$ and such that $\left(U_{i}, U_{i} \cap D\right)$ is homeomorphic either to $\left(R^{n}, H^{k}\right)$ or to $\left(R^{n}, R^{k}\right)$ for each $i$. Let $\left\{D_{1}, \cdots, D_{r}\right\}$ be a rectilinear subdivision of $D$ with the following properties:

(1) each $D_{i}$ is a $k$-cell piecewise linearly embedded in $D$,

(2) each $D_{i}$ is contained in some $U_{j}$,

(3) $E_{i}=D_{i} \cup \ldots \cup D_{r}$ is a $k$-cell for each $i$,

(4) $D_{i} \cap E_{i+1}=\dot{D}_{i} \cap \dot{E}_{i+1}$ is a $(k-1)$-cell piecewise linearly embedded in both $\dot{D}_{i}$ and $\dot{E}_{i+1}$.

For each $i=1, \cdots, r-1$ construct a homeomorphism $h_{i}$ of $M$ on to itself such that $h_{i}\left(E_{i}\right)=E_{i+1}$. Letting $h$ be the composition of the $h_{i}, h$ is a homeomorphism of $M$ which maps $D=E_{1}$ on to $D_{r}=E_{r}$. Since $D_{r}$ has a neighborhood of the desired type, the proof is complete.

Construction of $h_{1}$. From conditions (1) and (2) it follows that there is a $U_{j}$ and a homeomorphism $\phi_{1}: U_{j} \approx R^{n}$ such that $\phi_{1}\left(U_{j} \cap D\right)=H^{k}$ and $\phi_{1}\left(D_{1}\right)=B^{k} \cap H^{k}$. Let $\psi_{1}$ be a homeomorphism of $R^{n}$ which is the identity outside some compact set and which takes $H^{k}$ on to $H^{k}-\check{B}^{k}$. Define $h_{1}$ on $M$ by

$$
h_{1}= \begin{cases}\phi_{1}^{-1} \psi_{1} \phi_{1} & \text { on } U_{1}, \\ \text { identity } & \text { on } M-U_{1}\end{cases}
$$


Clearly $h_{1}$ takes $E_{1}$ onto $E_{2} . h_{2}, \cdots, h_{r-1}$ may be constructed in a similar manner.

\section{REFERENCES}

1. M. Brown, Lucally flat imbeddings of topological manifolds, Ann. of Math. (2) 75 (1962), 331-341.

2. - The monotone union of open n-cells is an open n-cell, Proc. Amer. Math. Soc. 12 (1961), 812-814.

3. J. C. Cantrell, Almost locally flat embeddings of $S^{n-1}$ in $S^{n}$, Bull. Amer. Math. Soc. 69 (1963), 716-718.

4. R. H. Fox, Some problems in knot theory, Topology of 3-Manifolds, PrenticeHall, Englewood Cliffs, New Jersey, 1962.

5. J. Stallings, On topologically unknotted spheres, Ann. of Math. 78 (1963), 501526.

6. R. Tindell, Some wild imbeddings in codimension two, Proc. Amer. Math. Soc. 17 (1966), 711-716.

UNIVERSITY OF GEORGIA 